\title{
Exact Solution to Nonlinear Differential Equations of Fractional Order via $\left(G^{\prime} / G\right)$-Expansion Method
}

\author{
Muhammad Younis, Asim Zafar \\ Centre for Undergraduate Studies, University of the Punjab, Lahore, Pakistan \\ Email: younis.pu@gmail.com, asimzafar@hotmail.com
}

Received August 16, 2013; revised September 16, 2013; accepted September 23, 2013

Copyright (C) 2014 Muhammad Younis, Asim Zafar. This is an open access article distributed under the Creative Commons Attribution License, which permits unrestricted use, distribution, and reproduction in any medium, provided the original work is properly cited. In accordance of the Creative Commons Attribution License all Copyrights (C) 2014 are reserved for SCIRP and the owner of the intellectual property Muhammad Younis, Asim Zafar. All Copyright (C) 2014 are guarded by law and by SCIRP as a guardian.

\section{ABSTRACT}

In this article, a new application to find the exact solutions of nonlinear partial time-space fractional differential Equation has been discussed. Firstly, the fractional complex transformation has been implemented to convert nonlinear partial fractional differential Equations into nonlinear ordinary differential Equations. Afterwards, the $\left(G^{\prime} / G\right)$-expansion method has been implemented, to celebrate the exact solutions of these Equations, in the sense of modified Riemann-Liouville derivative. As application, the exact solutions of time-space fractional Burgers' Equation have been discussed.

\section{KEYWORDS}

\section{Exact Solutions; Complex Transformation; $\left(G^{\prime} / G\right)$-Expansion Method; Nonlinear BURGERS' Equation; Fractional Calculus Theory}

\section{Introduction}

Nonlinear partial differential equations have shown a variety of applications in almost every field, such as in electromagnetic, acoustics, electrochemistry, cosmology, biological and material science [1-4]. Fractional differential equations can be considered as the generalization form of the differential equations, as they are involved with the derivatives of any real or complex order (for details see [3]).

In literature a lot of work has been done on nonlinear partial differential equations [5-8] rather than nonlinear partial differential equations (NPDEs) of fractional order. Recently, some new techniques have been introduced, by different authors, to find traveling wave solutions for NPDEs of fractional order [9-11].

In this article, the $\left(G^{\prime} / G\right)$-expansion method [5] has been applied in the sense of modified RiemannLiouville derivative to find the exact solutions of space-time fractional nonlinear Burgers' Equation, which has the following form [12]:

$$
\frac{\partial^{\alpha} u}{\partial t^{\alpha}}+\omega u \frac{\partial^{\beta} u}{\partial x^{\beta}}+\eta \frac{\partial^{2 \beta} u}{\partial x^{2 \beta}}=0, t>0,0<\alpha, \beta \leq 1,
$$

The rest of the letter is organized as follows, in Section 2 the basic definitions and properties of the fractional calculus are considered regarding to modified Riemann-Liouville derivative. In Section 3, the $\left(G^{\prime} / G\right)$-expansion method has been proposed to find the exact solutions for NPDEs of fractional order with the help of fractional complex transformation. As an application, the new exact solutions of nonlinear Burgers' Equation have been discussed in Section 4. In last Section 5, the conclusion has been drawn.

\section{Preliminaries and Basic Definitions}

In this section, the method has been applied in the sense of the Jumarie's modified Riemann-Liouville derivative 
$[13,14]$ of order $\alpha$. For this, some basic definitions and properties of the fractional calculus theory are being considered (for details see [3]). Thus, the fractional integral and derivatives can be defined following $[13,14]$ as:

Definition 2.1 A real function $f(s), s>0$, is said to be in the space $C_{\kappa}, \kappa \in R$, if there exists a real number $p>\kappa$ such that $f(s)=s^{p} f_{1}(s)$, where $f_{1}(s) \in C(0, \infty)$, and it is said to be in the space $C_{\kappa}^{m}$ if $f^{m} \in C_{\kappa}$, $m \in N$.

Definition 2.2 The Jumarie's modified Riemann-Liouville derivative, of order $\alpha$, can be defined by the following expression:

$$
D_{s}^{\alpha} f(s)= \begin{cases}\frac{1}{\Gamma(1-\alpha)} \frac{\mathrm{d}}{\mathrm{d} s} \int_{0}^{s}(s-\xi)^{-\alpha}(f(\xi)-f(0)) \mathrm{d} \xi, & 0<\alpha<1, \\ \left(f^{(n)}(s)\right)^{\alpha-n}, & n \leq \alpha<n+1, n \geq 1 .\end{cases}
$$

Moreover, some properties for the modified Riemann-Liouville derivative have also been given as follows:

$$
\begin{aligned}
& D_{s}^{\alpha} s^{r}=\frac{\Gamma(1+r)}{\Gamma(1+r-\alpha)} s^{r-\alpha}, \\
& D_{s}^{\alpha}(f(s) g(s))=f(s) D_{s}^{\alpha} g(s)+g(s) D_{s}^{\alpha} t(s), \\
& D_{s}^{\alpha} f[g(s)]=f_{g}^{\prime}[g(s)] D_{s}^{\alpha} g(s)=D_{s}^{\alpha} f[g(s)]\left(g^{\prime}(t)\right)^{\alpha} .
\end{aligned}
$$

\section{The $\left(G^{\prime} / G\right)$-Expansion Method for Nonlinear Fractional Partial Differential Equations}

In this section, the $\left(G^{\prime} / G\right)$-expansion method $[15,16]$ has been discussed to obtain the solutions of nonlinear fractional partial differential equations.

For this, we consider the following NPDE of fractional order:

$$
\begin{aligned}
& P\left(u, D_{t}^{\alpha} u, D_{s}^{\beta} u, D_{x}^{\gamma} u, \cdots, D_{t}^{\alpha} D_{t}^{\alpha} u, D_{t}^{\alpha} D_{s}^{\beta} u, D_{s}^{\beta} D_{s}^{\beta} u, D_{s}^{\beta} D_{x}^{\gamma} u, \cdots\right)=0, \\
& \text { for } 0<\alpha, \beta, \gamma<1,
\end{aligned}
$$

where $u$ is an unknown function and $P$ is a polynomial of $u$ and its partial fractional derivatives along with the involvement of higher order derivatives and nonlinear terms.

To find the exact solutions, the $\left(G^{\prime} / G\right)$-expansion method can be performed using the following steps.

Step 1: First, we convert the NFPDE into nonlinear ordinary differential Equations using fractional complex transformation introduced by Li et al. [17].

The travelling wave variable

$$
u(t, x, y)=u(\xi), \xi=\frac{K t^{\alpha}}{\Gamma(\alpha+1)}+\frac{L x^{\beta}}{\Gamma(\beta+1)}+\frac{M y^{\gamma}}{\Gamma(\gamma+1)}
$$

where $K, L$ and $M$ are non-zero arbitrary constants, permits us to reduce Equation (2) to an ODE of $u=u(\xi)$ in the following form

$$
P\left(u, u^{\prime}, u^{\prime \prime}, u^{\prime \prime \prime}, \cdots\right)=0 .
$$

If the possibility occurs, then Equation (4) can be integrated term by term one or more times.

Step 2: Suppose that the solution of Equation (4) can be expressed as a polynomial of $\left(G^{\prime} / G\right)$ in the form:

$$
u(\xi)=\sum_{i=-m}^{m} \alpha_{i}\left(\frac{G^{\prime}}{G}\right)^{i}, \alpha_{m} \neq 0,
$$

where $\alpha_{i^{\prime}}$ s are constants and $G(\xi)$ satisfies the following second order linear ordinary differential equation.

$$
G^{\prime \prime}(\xi)+\lambda G^{\prime}(\xi)+\mu G(\xi)=0
$$

with $\lambda$ and $\mu$ as constants.

Step 3: The homogeneous balance can be used, to determine the positive integer $m$, between the highest or- 
der derivatives and the nonlinear terms appearing in (4).

Moreover, the degree of $u(\xi)$ can be defined as $D[u(\xi)]=m$, which gives rise to the degree of the other expressions as follows:

$$
D\left[\frac{\mathrm{d}^{q} u}{\mathrm{~d} \xi q}\right]=m+q, D\left[u^{p}\left(\frac{\mathrm{d}^{q} u}{\mathrm{~d} \xi q}\right)^{s}\right]=m q+s(q+m) .
$$

Therefore, the value of $m$ can be obtained for the Equation (5).

Step 4: After the substitution of (5) into (4) and using Equation (6), we collect all the terms with the same order of $\left(G^{\prime} / G\right)$ together. Equate each coefficient of the obtained polynomial to zero, yields the set of algebraic equations for $K, L, M, \lambda, \mu$ and $\alpha_{i}(i=0, \pm 1, \pm 2, \cdots, \pm m)$.

Step 5: After solving the system of algebraic equations, and using the Equation (6), the variety of exact solutions can be constructed.

\section{Application of Burgers' Equation}

In this section, the improved $\left(G^{\prime} / G\right)$-expansion method have been used to construct the exact solutions for nonlinear space-time fractional Burgers' Equation (1).

$$
\frac{\partial^{\alpha} u}{\partial t^{\alpha}}+a u \frac{\partial^{\beta} u}{\partial x^{\beta}}+b \frac{\partial^{2 \beta} u}{\partial x^{2 \beta}}=0, t>0,0<\alpha, \beta \leq 1 .
$$

It can be observed that the fractional complex transform

$$
u(x, t)=u(\xi), \xi=\frac{K x^{\beta}}{\Gamma(\beta+1)}+\frac{L t^{\alpha}}{\Gamma(\alpha+1)}
$$

where $K$ and $L$ are constants, permits to reduce the Equation (7) into an ODE. After integrating once, we have the following form:

$$
C+L U+\frac{a}{2} K U^{2}+b K^{2} U^{\prime}=0
$$

where $C$ is a constant of integration. Now by considering the homogeneous balance between the highest order derivatives and nonlinear term presented in the above Equation, we have the following form

$$
u(\xi)=\alpha_{1}\left(\frac{G^{\prime}}{G}\right)+\alpha_{0}+\alpha_{-1}\left(\frac{G^{\prime}}{G}\right)^{-1}, \alpha_{1} \neq 0,
$$

where $\alpha_{-1}, \alpha_{0}, \alpha_{1}, K$ and $L$ are arbitrary constants. To determine these constants substitute Equation (10) into (9), and collecting all the terms with the same power of $\left(G^{\prime} / G\right)$ together, equating each coefficient equal to zero, yields a set of algebraic Equations.

$$
\begin{aligned}
& \frac{1}{2} a \alpha_{1}^{2} K-b K^{2} \alpha_{1}=0, \\
& L \alpha_{1}+a \alpha_{1} \alpha_{0} K-b K^{2} \lambda \alpha_{1}=0, \\
& C+L \alpha_{0}+\frac{1}{2} a K\left(\alpha_{0}^{2}+2 \alpha_{-1} \alpha_{1}\right)+b K^{2} \alpha_{-1}(1-\mu)=0, \\
& L \alpha_{-1}+a \alpha_{-1} \alpha_{0} K+b K^{2} \lambda \alpha_{-1}=0, \\
& \frac{1}{2} a \alpha_{-1}^{2} K+b \mu K^{2} \alpha_{-1}=0 .
\end{aligned}
$$

After solving these algebraic equations with the help of software Maple, yields the following results.

Case 1 For the values:

$$
K=\frac{\left(a^{2} C-b L^{2}\right)}{\lambda a b L}, \alpha_{1}=\frac{2 b K}{a}, \alpha_{0}=-\frac{L}{a K} \text { and } \alpha_{-1}=0 .
$$


Straightforward simplification of Equation (10) yields, the following equation

$$
u(\xi)=\frac{2 b K}{a}\left(\frac{G^{\prime}}{G}\right)-\frac{L}{a K} .
$$

From Equations (6) and (11), we have the following travelling wave solutions.

If $\lambda^{2}-4 \mu>0$, then we have the following hyperbolic solution

$$
u(\xi)=\frac{b K}{a} \sqrt{\lambda^{2}-4 \mu}\left(\frac{A \cosh \left(\frac{\xi}{2} \sqrt{\lambda^{2}-4 \mu}\right)+B \sinh \left(\frac{\xi}{2} \sqrt{\lambda^{2}-4 \mu}\right)}{A \sinh \left(\frac{\xi}{2} \sqrt{\lambda^{2}-4 \mu}\right)+B \cosh \left(\frac{\xi}{2} \sqrt{\lambda^{2}-4 \mu}\right)}\right)-\frac{L}{a K} .
$$

If $\lambda^{2}-4 \mu<0$, then we have the following trigonometric solution

$$
u(\xi)=\frac{b K}{a} \sqrt{4 \mu-\lambda^{2}}\left(\frac{-A \sin \left(\frac{\xi}{2} \sqrt{4 \mu-\lambda^{2}}\right)+B \cos \left(\frac{\xi}{2} \sqrt{4 \mu-\lambda^{2}}\right)}{A \cos \left(\frac{\xi}{2} \sqrt{4 \mu-\lambda^{2}}\right)+B \sin \left(\frac{\xi}{2} \sqrt{4 \mu-\lambda^{2}}\right)}\right)-\frac{L}{a K}
$$

and if $\lambda^{2}-4 \mu=0$, then we have the following solution

$$
u(\xi)=\frac{2 b K}{a}\left(\frac{B}{A+B \xi}\right)-\frac{L}{a K},
$$

where

$$
\xi=\frac{\left(a^{2} C-b L\right) x^{\beta}}{\lambda a b L \Gamma(\beta+1)}+\frac{L t^{\alpha}}{\Gamma(\alpha+1)} .
$$

Case 2 For the values:

$$
\begin{aligned}
& C=\frac{b K^{2}\left(-\mu \alpha_{0}^{2}+\lambda \alpha_{-1} \alpha_{0}-\alpha_{-1}^{2}+\mu \alpha_{-1}^{2}\right)}{\alpha_{-1}}, \alpha_{-1}=\frac{2 b \mu K}{a}, \\
& \alpha_{0}=-\frac{1}{a}\left(L+\lambda b K^{2}\right) \text { and } \alpha_{1}=0 .
\end{aligned}
$$

Straightforward simplification of Equation (10) yields, the following equation

$$
u(\xi)=-\frac{1}{a}\left(L+\lambda b K^{2}\right)+\frac{2 b \mu K}{a}\left(\frac{G^{\prime}}{G}\right)^{-1},
$$

From Equations (6) and (12), we have the following travelling wave solutions.

If $\lambda^{2}-4 \mu>0$, then we have the following hyperbolic solution

$$
u(\xi)=-\frac{1}{a}\left(L+\lambda b K^{2}\right)+\frac{b \mu K}{a}\left(\lambda^{2}-4 \mu\right)^{-\frac{1}{2}}\left(\frac{A \cosh \left(\frac{\xi}{2} \sqrt{\lambda^{2}-4 \mu}\right)+B \sinh \left(\frac{\xi}{2} \sqrt{\lambda^{2}-4 \mu}\right)}{A \sinh \left(\frac{\xi}{2} \sqrt{\lambda^{2}-4 \mu}\right)+B \cosh \left(\frac{\xi}{2} \sqrt{\lambda^{2}-4 \mu}\right)}\right)^{-1} .
$$

If $\lambda^{2}-4 \mu<0$, then we have the following trigonometric solution

$$
u(\xi)=-\frac{1}{a}\left(L+\lambda b K^{2}\right)+\frac{b \mu K}{a}\left(\lambda^{2}-4 \mu\right)^{-\frac{1}{2}}\left(\frac{-A \sin \left(\frac{\xi}{2} \sqrt{\lambda^{2}-4 \mu}\right)+B \cos \left(\frac{\xi}{2} \sqrt{\lambda^{2}-4 \mu}\right)}{A \cos \left(\frac{\xi}{2} \sqrt{\lambda^{2}-4 \mu}\right)+B \sin \left(\frac{\xi}{2} \sqrt{\lambda^{2}-4 \mu}\right)}\right)^{-1}
$$


and if $\lambda^{2}-4 \mu=0$, then we have the following solution

$$
u(\xi)=-\frac{1}{a}\left(L+\lambda b K^{2}\right)+\frac{2 b \mu K}{a}\left(\frac{B}{A+B \xi}\right)^{-1},
$$

where

$$
\xi=\frac{K x^{\beta}}{\Gamma(\beta+1)}+\frac{L t^{\alpha}}{\Gamma(\alpha+1)}
$$

where $A$ and $B$ are arbitrary constants.

\section{Conclusion}

The $\left(G^{\prime} / G\right)$-expansion method has been extended to solve the nonlinear partial differential equation of fractional order, in the sense of modified Riemann-Liouville derivative. First, the fractional complex transformation has been used to convert the fractional equations into ordinary differential equation. Then $\left(G^{\prime} / G\right)$-expansion method has been used to find exact solutions. As an application, the new exact solutions for the space-time fractional Burgers' Equations have been found. It can be concluded that this method is very simple, reliable and proposes a variety of exact solutions to NPDEs of fractional order.

\section{REFERENCES}

[1] R. S. Johnson, “A Non-Linear Equation Incorporating Damping and Dispersion,” Journal of Fluid Mechanics, Vol. 42, No. 1, 1970, pp. 49-60. http://dx.doi.org/10.1017/S0022112070001064

[2] W. G. Glöckle and T. F. Nonnenmacher, "A Fractional Calculus Approach to Self-Similar Protein Dynamics," Biophysical Journal, Vol. 68, No. 1, 1995, pp. 46-53. http://dx.doi.org/10.1016/S0006-3495(95)80157-8

[3] I. Podlubny, "Fractional Differential Equations," Academic Press, San Diego, 1999.

[4] J. H. He, "Some Applications of Nonlinear Fractional Differential Equations and Their Applications," Bulletin of Science, Technology \& Society, Vol. 15, No. 2, 1999, pp. 86-90.

[5] M. Wang, X. Li and J. Zhang, "The $\left(G^{\prime} / G\right)$-Expansion Method and Travelling Wave Soltions of Nonlinear Evolution Equations in Mathematical Physics," Physical Letter A, Vol. 372, 2008, pp. 417-423. http://dx.doi.org/10.1016/j.physleta.2007.07.051

[6] Z. Feng, "On Explicit Excact Solutions to the Compound Burgers-KdV Equation," Physical Letter A, Vol. 293, No. 1-2, 2002, pp. 57-66. http://dx.doi.org/10.1016/S0375-9601(01)00825-8

[7] S. K. Liu, Z. T. Fu, S. D. Liu and Q. Zhao, "Jacobi Elliptic Function Expansion Method and Periodic Wave Solutions of Nonlinear Wave Equations,” Physical Letter A, Vol. 289, No. 1-2, 2001, pp. 69-74. http://dx.doi.org/10.1016/S0375-9601(01)00580-1

[8] M. Younis and A. Zafar, "The Modified Simple Equation Method for Solving Nonlinear Phi-Four Equation," International Journal of Innovation and Applied Studies, Vol. 2 No. 4, 2013, pp. 661-664.

[9] K. A. Gepreel, "The Homotopy Perturbation Method Applied to the Nonlinear Fractional Kolmogorov Petrovskii Piskunov Equations," Applied Mathematics Letters, Vol. 24, No. 8, 2011, pp. 1428-1434. http://dx.doi.org/10.1016/j.aml.2011.03.025

[10] G.-C. Wu, "A Fractional Characteristic Method for Solving Fractional Partial Differential Equations," Applied Mathematics Letters, Vol. 24, No. 7, 2011, pp. 1046-1050. http://dx.doi.org/10.1016/j.aml.2011.01.020

[11] M. Younis, “The First Integral Method for Time-Space Fractional Differential Equations," Journal of Advanced Physics, Vol. 2, No. 3, 2013, pp. 220-223. http://dx.doi.org/10.1166/jap.2013.1074

[12] Q. Wang, "Numerical Solutions for Fractional KDV-Burgers Equation by Adomian Decomposition Method," Applied Mathematics and Computation, Vol. 182, No. 2, 2006, pp. 1048-1055. http://dx.doi.org/10.1016/j.amc.2006.05.004

[13] G. Jumarie, "Modified Riemann-Liouville Derivative and Fractional Taylor Series of Nondifferentiable Functions Further Results," Computers \& Mathematics with Applications, Vol. 51, No. 9-10, 2006, pp. 1367-1624. http://dx.doi.org/10.1016/j.camwa.2006.02.001

[14] G. Jumarie, "Laplaces Transform of Fractional Order via the Mittag Leffler Function and Modified Riemann-Liouville Derivative," Applied Mathematics Letters, Vol. 22, No. 11, 2009, pp. 1659-1664. http://dx.doi.org/10.1016/j.aml.2009.05.011

[15] M. Younis, A. Zafar, K. Ul-Haq and M. Rahman, “Travelling Wave Solutions of Fractional Order Coupled Burger's Equation by $\left(G^{\prime} / G\right)$-Expansion Method," American Journal of Computational and Applied Mathematics, Vol. 3, No. 2, 2013 , pp. 81-85.

[16] K. A. Gepreel and S. Omran, "Exact Solutions for Nonlinear Partial Fractioanl Differential Equations," Chinese Physics B, Vol. 
21, No. 11, 2012, Article DI: 110204.

[17] Z.-B. Li and J.-H. He, "Fractional Complex Transform for Fractional Differential Equations," Computers \& Mathematics with Applications, Vol. 15, No. 5, 2010, pp. 970-973. 